\title{
Commentary on "Occlusion-Fit V-Line Guide and Gooseneck Saw for Safe and Accurate Mandibuloplasty in Asian [Patients]" by Song et al.
}

\author{
S. Anthony Wolfe ${ }^{1}$ (B)
}

Received: 10 July 2017 / Accepted: 12 July 2017/Published online: 4 August 2017

(c) Springer Science+Business Media, LLC and International Society of Aesthetic Plastic Surgery 2017

No Level Assigned This journal requires that authors assign a level of evidence to each article. For a full description of these Evidence-Based Medicine ratings, please refer to the Table of Contents or the online Instructions to Authors www.springer.com/00266.

The authors show that precise mandibular osteotomies can be obtained by making cutting guides based on CT data. They rightly point out that those benefiting the most from this new technology would be beginning surgeons; those with greater experience can probably obtain the same precise results without the need for the cutting guides and the added expense of a CT scan.

The greatest value of this technology I would think would be if the three-dimensional CT scan could show in another color the course of the inferior alveolar nerve, which can vary considerably in its vertical position.

The simple procedures such as a trim of the gonial angle and inferior mandibular border probably do not need cutting guides in experienced hands. The more complex double-jaw orthognathic procedures are the situations where virtual surgery and cutting guides are becoming standard of care.

\section{Compliance with Ethical Standards}

Conflict of interest The author declares that he has no conflict of interest.
S. Anthony Wolfe

drwolfe@santhonywolfemd.com

1 Division of Plastic Surgery, Miami Children's Health System, 3100 S.W. 62nd Avenue, Miami, FL 33155, USA 